\title{
Simultaneous measurement of strain and temperature based on clover microstructured fiber loop mirror
}

\author{
R.A. Pérez-Herrera ${ }^{\text {a,* }}$, R.M. André ${ }^{\mathrm{b}}$, S.F. Silva ${ }^{\mathrm{b}}$, M. Becker ${ }^{\mathrm{c}}$, K. Schuster ${ }^{\mathrm{c}}$, J. Kobelke ${ }^{\mathrm{c}}$, \\ M. Lopez-Amo ${ }^{\text {a }}$, J.L. Santos ${ }^{\text {b }}$, O. Frazão ${ }^{\text {b }}$ \\ a Public University of Navarra, Dept. of Electric and Electronic Engineering, Campus de Arrosadía, Pamplona, Spain \\ ' INESC Porto, Rua do Campo alegre, 687 Porto, Portugal \\ ${ }^{\mathrm{c}}$ Leibniz Institute of Photonic Technology, Albert-Einstein-Strasse 9, 07745 Jena, Germany
}

\section{A R T I C L E I N F O}

\section{Article history:}

Received 3 February 2014

Received in revised form 24 December 2014

Accepted 30 December 2014

Available online 12 January 2015

\section{Keywords:}

Interferometers

Fiber optic sensors

Multimode interference

Photonic crystal fibers

\begin{abstract}
A B S T R A C T
In this work, two all-fiber loop mirrors using a clover microstructured fiber for the simultaneous measurement of temperature and strain are presented. The sensing heads are formed by a short piece of clover microstructured fiber with $35 \mathrm{~mm}$ and $89 \mathrm{~mm}$ length respectively. The geometry of the fiber allowed observing different interferences created by the microstructured fiber core section. Different sensitivities to temperature and strain were obtained and, using a matrix method, it is possible to discriminate both physical parameters. Resolutions of $\pm 2{ }^{\circ} \mathrm{C}$ and $\pm 11 \mu \varepsilon$ for the first structure and $\pm 2.3^{\circ} \mathrm{C}$ and $\pm 18 \mu \varepsilon$ for the second one, for temperature and strain, respectively, were attained.
\end{abstract}

(c) 2015 Elsevier Ltd. All rights reserved.

\section{Introduction}

Fiber Loop Mirrors (FLMs) are very attractive structures to be used in several applications such as wavelength filters and sensors [1-3]. In a FLM, two interfering waves counter-propagate through the same fiber and are exposed to the same environment. FLMs made of highly birefringent fiber (HiBi-FLM) have several advantages, including input polarization independence and high extinction ratio. Besides the gyroscope application, various kinds of sensors based on FLMs have been realized [3], such as temperature sensors $[2,4]$, strain sensors $[5,6]$, pressure sensors, liquid level sensors [7], biochemical sensors, UV detection [8] and multiparameter measurement [9-11].

On the other hand, optical fiber sensors based on intermodal interference between core and cladding modes have some interesting features when compared with other

\footnotetext{
* Corresponding author.

E-mail address: rosa.perez@unavarra.es (R.A. Pérez-Herrera).
}

sensing concepts, including small size, fast response time, high resolution and sensitivity, and low cost $[12,13]$. Different solutions to obtain all-fiber intermodal interference in microstructured fibers are reported in published literature such as using a polarization maintaining fiber $[14,15]$ or a Mach-Zehnder interferometer [16] among others. In particular, the combination of the FLM topological layout with the multimodal interferometric sensing concept is an attractive approach which was the focus of the research presented here.

In this work, two different lengths of clover microstructured fibers with a central rectangular silica region containing an air hole defect have been inserted into a fiber loop mirror configuration. Owing to the use of this special clover microstructured fiber as transducer, four distinct interferometric contributions have been generated, associated with the presence of two embedded cores combined with birefringence. The use of this configuration allows the simultaneous characterization of the system both in strain and temperature, showing different sensitivities. 


\section{Experimental setup}

Fig. 1 shows the experimental setup containing a broadband light source (BBS), a fiber loop mirror (FLM) and an Optical Spectrum Analyzer (OSA) with $0.1 \mathrm{~nm}$ resolution. The broadband light used to illuminate the sensing head has $100 \mathrm{~nm}$ bandwidth at the $1550 \mathrm{~nm}$ window.

The first FLM was formed by a $3 \mathrm{~dB}$ optical coupler with low insertion loss and a small clover microstructured fiber section with $35 \mathrm{~mm}$ length that was spliced in the two output ports [17]. Two polarization controllers (PC1 and PC2) were also applied to control the interference obtained by the fiber. For the second FLM, a clover microstructured fiber section with $89 \mathrm{~mm}$ length was used.

The clover microstructured fiber, with a diameter of $\sim 200 \mu \mathrm{m}$, was fabricated at IPHT and presents four holes, each one with a diameter of about $\sim 30 \mu \mathrm{m}$. Defined by these holes there is a central silica region, approximately rectangular $\left(\sim 15.2 \times 11.82 \mu \mathrm{m}^{2}\right)$, with an inside rectangular air-hole with dimensions of $\sim 6.76 \times 3.38 \mu \mathrm{m}^{2}$. This air feature has a size that turns it more than a defect in the light propagation along the silica region, fairly splitting it in two cores. This was confirmed by a theoretical analysis of the light propagation in the structure, as shown in Fig. 1(b). Indeed, the light field is mostly concentrated in these cores, with some variation depending on the polarization of light. Therefore, this guiding structure exhibits birefringence. The consequence of these features when the fiber is inserted in the loop mirror is a birefringence interferometer associated with each core, i.e., the presence of four distinct interferometric contributions for the output signal.

\section{Experimental results}

Fig. 2 illustrates the spectral response of the first fiber loop sensor structure. As expected, it is a complex one in face of the several interference terms, originating beating phenomena. The fringes identified in Fig. 2 as $\lambda_{L}$ are associated with the standard fiber loop mirror channeled spectrum, while $\lambda_{B}$ is attributed to birefringence effects. Indeed, it was calculated this microstructured fiber to have a birefringence of $\sim 10^{-4}$ and, due to the reduced fiber length, only one peak is observed in the wavelength range $1500-1600 \mathrm{~nm}$. Also, it was observed the feature $\lambda_{B}$ be highly sensitive to the polarization state of the light. The presence of two cores introduces the extra degrees of freedom responsible for the additional beating effects observed in Fig. 2.

Both structures were characterized in strain at room temperature and also in temperature when no strain is applied. Fig. 3 shows the spectral shift of $\lambda_{L}$ and $\lambda_{B}$ when the first structure is subjected to strain. The result shows different linear responses of $\left(\lambda_{B}\right)$ and $\left(\lambda_{L}\right)$ with slope sensitivities of $-1.78 \mathrm{pm} / \mu \varepsilon$ and $-1.49 \mathrm{pm} / \mu \varepsilon$, respectively. The photo-elastic effect is dominant then a negative response was observed.

Fig. 4 shows the temperature response of the first structure and a linear behavior was also obtained. The sensitivities are $38.4 \mathrm{pm} /{ }^{\circ} \mathrm{C}$ and $6.6 \mathrm{pm} /{ }^{\circ} \mathrm{C}$ for $\left(\lambda_{L}\right)$ and $\left(\lambda_{B}\right)$ respectively. A positive response is observed due to high dependence of the thermal expansion coefficient (pure silica).

In order to sense the strain change $\Delta \varepsilon$ and temperature change $\Delta T$, independent and simultaneously, the wavelength shifts $\Delta \lambda_{B}$ and $\Delta \lambda_{L}$, were recorded by an Optical Spectrum Analyzer (OSA). These quantities can be expressed in terms of the strain change $\Delta \varepsilon$ and temperature change $\Delta T$ as follows:

$\Delta \lambda_{i}(\varepsilon, T)=K_{\varepsilon i} \Delta \varepsilon+K_{T i} \Delta T$

where $i=B, L$. One can observe that $K_{T}$ and $K_{\varepsilon}$ are distinct when monitoring $\lambda_{B}$ or $\lambda_{L}$, enabling the simultaneous measurement methodology which yields two linear equations whose matrix form is:

$\left[\begin{array}{l}\Delta \lambda_{B} \\ \Delta \lambda_{L}\end{array}\right]=\left[\begin{array}{ll}K_{\varepsilon B} & K_{T B} \\ K_{\varepsilon L} & K_{T L}\end{array}\right]\left[\begin{array}{l}\Delta \varepsilon \\ \Delta T\end{array}\right]$

where $K_{\varepsilon B}, K_{\varepsilon L}$ and $K_{T B}, K_{T L}$ are respectively the strain and temperature sensitivities associated with $\lambda_{B}$ and $\lambda_{L}$. In order to efficiently discriminate the temperature and the strain contributions, the matrix of coefficients $K$ must be well conditioned. The relative values $\Delta \varepsilon$ and $\Delta T$ are obtained by the following equation:

$\left[\begin{array}{l}\Delta \varepsilon \\ \Delta T\end{array}\right]=\frac{1}{45.468}\left[\begin{array}{ll}6.6 & -38.4 \\ 1.49 & -1.78\end{array}\right]\left[\begin{array}{l}\Delta \lambda_{B} \\ \Delta \lambda_{L}\end{array}\right]$

where the wavelength shifts $\Delta \lambda_{L}$ and $\Delta \lambda_{B}$ are expressed in picometers $(\mathrm{pm})$, the strain variation $(\Delta \varepsilon)$ in microstrain $(\mu \varepsilon)$ and the temperature variation $(\Delta T)$ in Celsius degrees $\left({ }^{\circ} \mathrm{C}\right)$.

When the spread of the data shown in Figs. 3 and 4 is taken into consideration for the evaluation of the slopes (sensitivities) uncertainties, it turns out fluctuations with

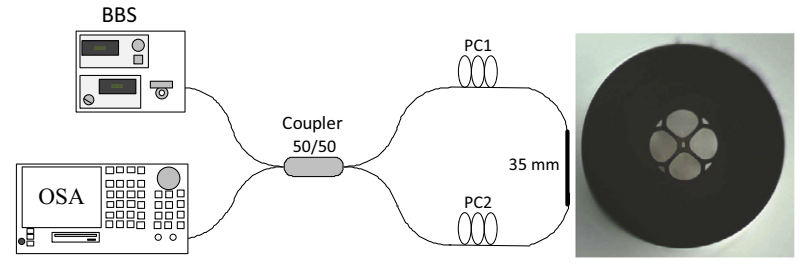

(a)
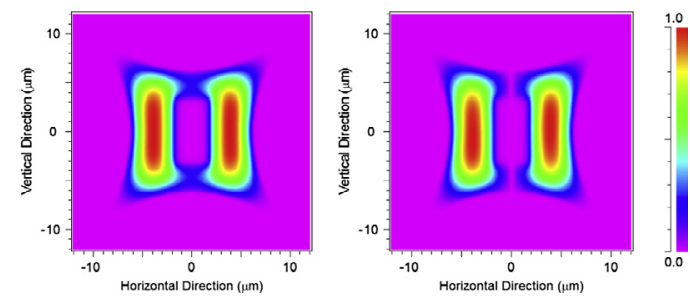

(b)

Fig. 1. (a) Schematic diagram of the sensing system (a photograph of the clover microstructured fiber is also shown). (b) Modeling of the light propagation in the microstructured fiber for two orthogonal polarizations. 

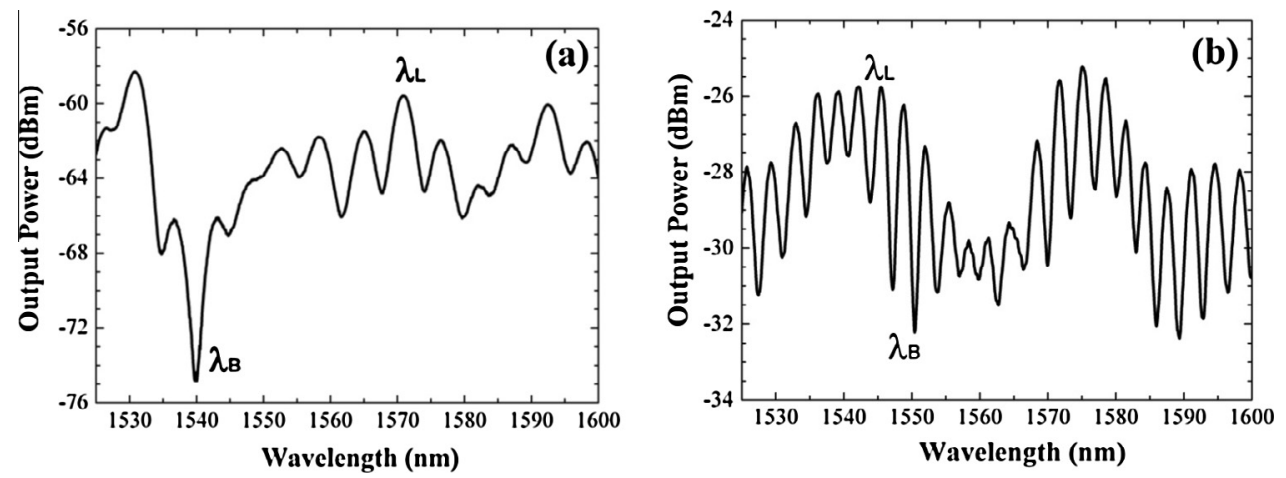

Fig. 2. Output spectrum response of the fiber loop sensing structure using a clover microstructured fiber section with (a) 35 mm or (b) 89 mm length.

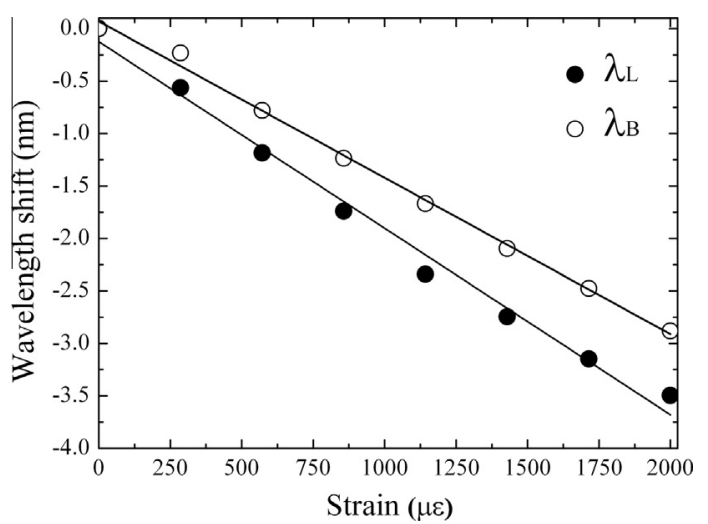

Fig. 3. Wavelength shift as a function of strain change for the wavelengths $\lambda_{L}$ and $\lambda_{B}$ (first structure).

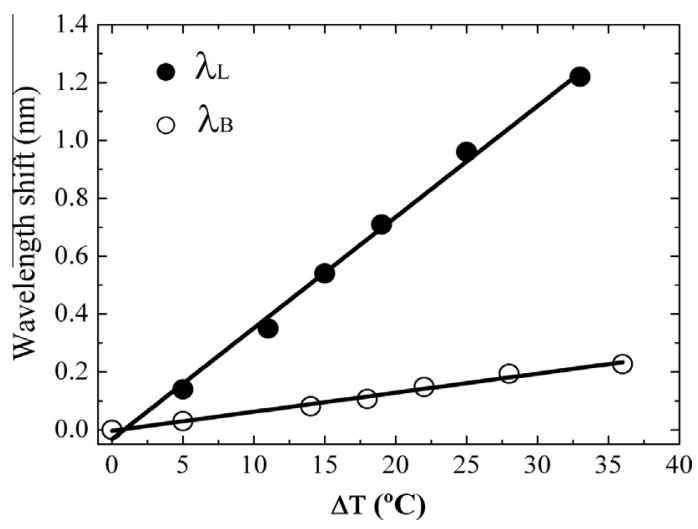

Fig. 4. Wavelength shift as a function of temperature change for the wavelengths $\lambda_{L}$ and $\lambda_{B}$ (first structure).

rms amplitudes of $\pm 2{ }^{\circ} \mathrm{C}$ and $\pm 11 \mu \varepsilon$ for temperature and strain, respectively, which can be considered the resolutions obtained with this structure when implementing measurement of these two parameters simultaneously.

This process was also carried out for the second structure, the one with a clover microstructured fiber section with $89 \mathrm{~mm}$. Fig. 5 shows the spectral shift of $\lambda_{L}$ and $\lambda_{B}$

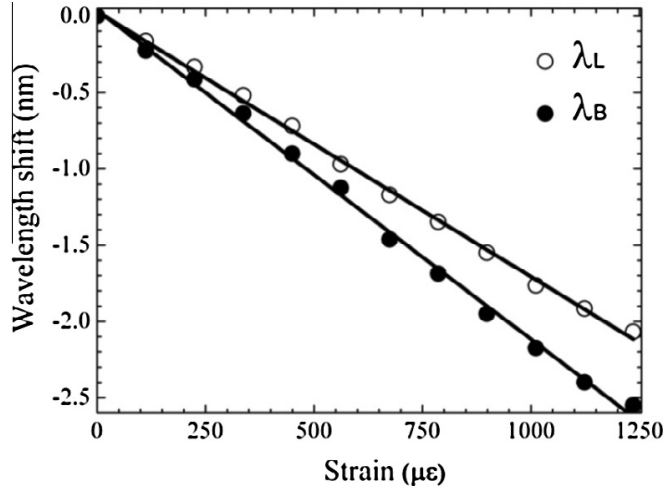

Fig. 5. Wavelength shift as a function of strain change for the wavelengths $\lambda_{L}$ and $\lambda_{B}$ (second structure).

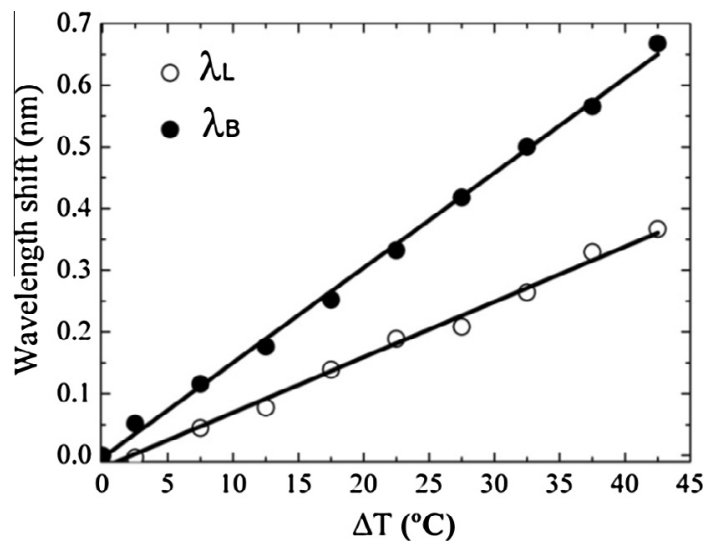

Fig. 6. Wavelength shift as a function of temperature change for the wavelengths $\lambda_{L}$ and $\lambda_{B}$ (second structure).

when the second structure is subjected to strain. Once again the result shows different linear responses of $\left(\lambda_{B}\right)$ and $\left(\lambda_{L}\right)$ with slope sensitivities of $-2.12 \mathrm{pm} / \mu \varepsilon$ and $-1.74 \mathrm{pm} / \mu \varepsilon$, respectively. On the other hand, Fig. 6 shows the temperature response of the second structure and again a linear behavior was obtained. The sensitivities are $15.37 \mathrm{pm} /{ }^{\circ} \mathrm{C}$ and $8.95 \mathrm{pm} /{ }^{\circ} \mathrm{C}$ for $\left(\lambda_{B}\right)$ and $\left(\lambda_{L}\right)$ respectively. 
In this case, the relative values $\Delta \varepsilon$ and $\Delta T$ are obtained by the following equation:

$$
\left[\begin{array}{l}
\Delta \varepsilon \\
\Delta T
\end{array}\right]=\frac{1}{7.77}\left[\begin{array}{ll}
8.95 & -15.37 \\
1.74 & -2.12
\end{array}\right]\left[\begin{array}{l}
\Delta \lambda_{B} \\
\Delta \lambda_{L}
\end{array}\right]
$$

In this case, the root-mean-square deviations relative to the applied values are $\pm 2.3^{\circ} \mathrm{C}$ and $\pm 18 \mu \varepsilon$ for temperature and strain, respectively.

Comparing these values with those derived from a smaller length of clover microstructured fiber, it seems the shorter option is favorable from the measurand resolution side, which goes in line with a better readout stability and sensor compactness. But eventually most relevant to emphasize is what results from the combination of the configuration fiber loop mirror with modal interferometry implemented with microstructured optical fibers, i.e. a large level of design flexibility permitting to tailor the sensor operation to specific requirements associated with a targeted application. This is always an added value characteristic emphasizing the attractiveness of this fiber optic sensing head proposal.

\section{Conclusions}

In conclusion, two different all-fiber loop mirrors using a clover microstructured fiber for simultaneous measurement of temperature and strain have been reported. The clover fiber has a rectangular core with an air-hole defect and this structure in the ring layout originates interference features that show different sensitivities to strain and temperature. Applying the matrix method it was possible to discriminate both parameters. For the small length sensor, resolutions of $\pm 2{ }^{\circ} \mathrm{C}$ and $\pm 11 \mu \varepsilon$ for temperature and strain were obtained, values that become $\pm 2.3^{\circ} \mathrm{C}$ and $\pm 18 \mu \varepsilon$ for the sensing structure with higher length of microstructured fiber. Therefore, from the resolution side the consideration of a small length of sensing fiber is advantageous; the same can be said when addressing the sensor readout stability and compactness.

The proposed sensing head combines features which result from the fiber loop mirror layout with those associated with modal interferometry supported by special fibers. This means added degrees of freedom are present which permits the tailoring of structures of this type to specific sensing requirements.

\section{Acknowledgments}

This work was supported in part by the European COST action TD1001, FEDER founds and the Spanish Government project TEC2013-47264-C2-2-R1.

\section{References}

[1] D.B. Mortimore, Fiber loop reflectors, J. Lightwave Technol. 6 (1988) $1217-1224$.

[2] Y. Wang, C.-L. Zhao, X. Dong, J. Kang, S. Jin, A fiber loop mirror temperature sensor demodulation technique using a long-period grating in a photonic crystal fiber and a band-pass filter, Rev. Sci. Instrum. 82 (2011) 073101 (5pp).

[3] Y. Liu, B. Liu, X. Feng, W. Zhang, G. Zhou, S. Yuan, G. Kai, X. Dong, High-birefringence fiber loop mirrors and their applications as sensors, Appl. Opt. 44 (2005) 2382-2390.

[4] C.-L. Zhao, X. Yang, C. Lu, W. Jin, M.S. Demokan, Temperatureinsensitive interferometer using a highly birefringent photonic crystal fiber loop mirror, IEEE Photon. Technol. Lett. 16 (2004) 2535-2537

[5] M. Campbell, G. Zheng, A.S. Holmes-Smith, P.A. Wallace, A frequency-modulated continuous wave birefringent fiber-optic strain sensor based on a Sagnac ring configuration, Meas. Sci. Technol. 10 (1999) 218-224.

[6] A.M. Hatta, Y. Semenova, Q. Wu, G. Farrell, Strain sensor based on a pair of single-mode-multimode-single-mode fiber structures in a ratiometric power measurement scheme, Appl. Opt. 49 (2010) 536541.

[7] B. Dong, Q. Zhao, F. Lvjun, T. Guo, L. Xue, S. Li, H. Gu, Liquid-level sensor with a high-birefringence-fiber loop mirror, Appl. Opt. 45 (2006) 7767-7771.

[8] K.-J. Kim, S.-H. Yeom, B.-H. Kang, D.-E. Kim, S.-W. Kang, Micro-optic Mach-Zehnder interferometric sensor for UV detection using photochromic dye, Sens. Lett. 9 (2011) 195-198.

[9] S. Silva, O. Frazão, J. Viegas, L.A. Ferreira, F.M. Araújo, F.X. Malcata, J.L. Santos, Temperature and strain-independent curvature sensor based on a singlemode/multimode fiber optic structure, Meas. Sci. Technol. 22 (2011) 085201 (6pp).

[10] R.M. André, M.B. Marques, P. Roy, O. Frazão, Fiber loop mirror using a small core microstructured fiber for strain and temperature discrimination, IEEE Photonics Technol. Lett. 22 (2010) 1120-1122.

[11] D.-P. Zhou, L. Wei, W.-K. Liu, Y. Liu, J.W.Y. Lit, Simultaneous measurement for strain and temperature using fiber Bragg gratings and multimode fibers, Appl. Opt. 47 (2008) 1668-1672.

[12] G. Statkiewicz-Barabach, J.P. Carvalho, O. Frazão, J. Olszewski, P. Mergo, J.L. Santos, W. Urbanczyk, Intermodal interferometer for strain and temperature sensing fabricated in birefringent boron doped microstructured fiber, Appl. Opt. 50 (2011) 3742-3749.

[13] S. Rota-Rodrigo, S. López-Amo, J. Kobelke, K. Schuster, J.L. Santos, O. Frazão, Multimodal interferometer based on a suspended core fiber for simultaneous measurement of physical parameters, J. Lightwave Technol. (2014) doi:http://dx.doi.org/10.1109/JLT.2014.2375896 (in press).

[14] J. Chu, Ch. Shen, F. Qian, Ch. Zhong, X. Zou, X. Dong, Y. Jin, J. Wang, Y Gong, T. Jiang, Simultaneous measurement of strain and temperature based on a long-period grating with a polarization maintaining fiber in a loop mirror, Opt. Fiber Technol. 20 (2014) 4447.

[15] Q. Wang, J. Xia, Y. Zhao, X. Liu, P. Wang, Y.-N. Zhang, C. Sang, L. Wang, Simultaneous measurement of strain and temperature with polarization maintaining fiber Bragg grating loop mirror, Instrum Sci. Technol. 42 (2014) 298-307.

[16] J. Zhou, C. Liao, Y. Wang, G. Yin, X. Zhong, K. Yang, B. Sun, G. Wang, Z. $\mathrm{Li}$, Simultaneous measurement of strain and temperature by employing fiber Mach-Zehnder interferometer, Opt. Exp. 22 (2014) 1680-1686.

[17] R.A. Perez-Herrera, R.M. André, S.F. Silva, M. Becker, K. Schuster, J. Kobelke, M. Lopez-Amo, J.L. Santos, O. Frazão, Simultaneous measurement of strain and temperature based on clover microstructured fiber loop mirror, in: Proceedings of the SPIE 8421 84216W 22nd International Conference on Optical Fiber Sensors (OFS-22). Proc. SPIE, OFS2012 22nd International Conference on Optical Fiber Sensors Beijing China, 15-19 October 2012. 